\title{
Use of silenced plants in allelopathy bioassays: a novel approach
}

\author{
Inderjit · Caroline C. von Dahl · Ian T. Baldwin
}

Received: 30 September 2008 / Accepted: 9 November 2008 / Published online: 26 November 2008

(C) The Author(s) 2008. This article is published with open access at Springerlink.com

\begin{abstract}
Volatile phytohormones or other chemicals can affect processes in distal plant parts but may also influence neighboring plants, and thereby function allelopathically. While this hypothesis has been widely discussed, rigorous tests are lacking. Transgenic plants, silenced in the production of an emitted chemical, are ideal tools to test the hypothesis that the release of a chemical can negatively influence the growth of neighbors (allelopathy). We used isogenic wild type (WT) and genetically transformed plants that lacked the ability to produce ethylene (ir- $a c o$ ), as both "emitters" and "receivers" of this volatile phytohormone in experiments where receiver plants were only exposed to the headspace of WT or ir-aco emitters, in order to evaluate if natural ethylene releases can function allelopathically. Root growth (a proxy of plant fitness) of WT receivers correlated negatively with the number of WT emitters and headspace ethylene concentrations. Reducing ethylene concentrations
\end{abstract}

Electronic supplementary material The online version of this article (doi:10.1007/s00425-008-0856-4) contains supplementary material, which is available to authorized users.

Inderjit

Centre for Environmental Management of Degraded Ecosystems (CEMDE), University of Delhi, Delhi 110007, India

e-mail: inderjit@cemde.du.ac.in

C. C. von Dahl

Boyce Thompson Institute for Plant Research,

Tower Road, Ithaca, NY 14853, USA

e-mail: cv75@cornell.edu

\section{T. Baldwin ( $\square)$}

Department of Molecular Ecology,

Max-Planck-Institute for Chemical Ecology,

Hans-Knöll-Strasse 8, Jena 07745, Germany

e-mail: baldwin@ice.mpg.de in the headspace with the ethylene scrubber, $\mathrm{KMnO}_{4}$, and using ir-aco seedlings as emitters restored root growth of WT receiver seedlings. 1-Aminocyclopropane-1-carboxylic acid (ethylene biosynthesis substrate) supplementation to WT but not ir-aco emitters inhibited root growth of ir-aco, but not WT receivers, suggesting increased sensitivity to exogenous ethylene of ir-aco seedlings. We conclude that plants genetically silenced in the production of a putative allelochemical are useful in determining if the emitted chemical functions allelopathically.

Keywords Allelopathy - Ethylene - Nicotiana attenuate . Growth inhibition · Plant-plant interaction $\cdot$ Phytohormone

$\begin{array}{ll}\text { Abbreviations } \\ \text { ACC } & 1 \text {-Aminocyclopropane-1-carboxylic acid } \\ \text { ACO } & \text { ACC oxidase } \\ \text { ACS } & \text { ACC synthase } \\ \text { SAM } & S \text {-adenosyl-L-methionine } \\ \text { WT } & \text { Wild type }\end{array}$

\section{Introduction}

Neighboring and competing plants can influence each other's growth indirectly by removing nutrients, light and other resources, but also directly by releasing compounds that inhibit growth and reproductive performance. Growth inhibition by volatile compounds of neighboring plants (allelopathy) has yet to be conclusively proven due to lack of direct evidence (Inderjit et al. 2006; Karban 2007). Transgenic plants, silenced for the production of a volatile chemical or phytohormone, represent ideal tools to examine the putative allelopathic function of emitted chemicals (Baldwin 2003). Similar approaches have been used to 
demonstrate the role of plant-produced secondary metabolites in plant-herbivore and other plant-plant interactions (Baldwin et al. 2006). By transforming plants to alter their ability to synthesize and release specific compounds and using such plants as "emitters" or "receivers" in growth experiments can resolve the question whether changes in growth and performance of neighboring plants can be attributed to a plant's release of a phytohormone (Baldwin 2003; Baldwin et al. 2006).

Ethylene is an important volatile phytohormone (Kepczynski 1986; Foo et al. 2006) that was discovered early in the century (Gane 1934). Whether the release of this volatile phytohormone can influence the growth of neighboring plants is not yet resolved. Pierik et al. (2003) used transformed cultivated tobacco expressing a mutant ethylene receptor (etrl-1) to demonstrate that ethylene signaling was required for cultivated tobacco to compete with its neighbors and elegantly showed in subsequent work, that exposure to ethylene elicited shade-avoidance growth responses (Pierik et al. 2004). The mechanisms of ethylene biosynthesis, specifically, the two major rate-limiting steps in ethylene biosynthesis, the conversion of $S$-adenosyl-Lmethionine (SAM) to 1-aminocyclopropane-1-carboxylic acid (ACC) by ACC synthase (ACS), and its subsequent oxidation to ethylene by ACC oxidase (ACO), as well as the family of ethylene receptors that the plant uses to sense ethylene, are well described molecularly (Wang et al. 2002; Klee 2004). As a consequence, both ethylene production and perception have been successfully manipulated by transforming plants to alter the expression of these molecularly defined targets (Hamilton et al. 1990; Knoester et al. 1998).

In order to understand the role of ethylene in plant-herbivore interactions, von Dahl et al. (2007) silenced ethylene biosynthesis in Nicotiana attenuata, a wild relative of cultivated tobacco ( $N$. tabacum) by transformation with an RNAi construct harboring a fragment of the plant's ACO in an inverted repeat orientation (ir-aco). Here we use this $N$. attenuata transformant, ir-aco, along with isogenic wild type (WT) plants in experiments where receiver plants were only exposed to the headspace of WT or ir-aco emitters in order to evaluate if natural ethylene releases can function allelopathically.

\section{Materials and methods}

Plant growth material

Seeds of an inbred line of $N$. attenuata Torr. ex. Wats. (synonymous with Nicotiana torreyana Nelson and Macbr.) as well as of the genetically transformed inverted repeat (ir) ir-aco line, silenced in the expression of the ethylene biosynthesis gene ACO (A03-321-10 as described in von Dahl et al. (2007) were germinated on Gamborg's B5 medium as described in Krügel et al. (2002).

\section{Seedling arena and experiments}

All seedling assays were performed in a growth arena composed of a two- or three-chamber Petri dish design (Supplementary Figure 1). Seedlings were germinated directly in the experimental setup. In all experiments, five seedlings (receivers) were grown in an open 6-cm Petri dish containing $20 \mathrm{ml}$ Gamborg's B5 medium. We selected a fixed density of five seeds for receivers because ethylene emission at the density of five seedlings did not differ from the background emission. The 6-cm Petri dish was placed in the middle of a larger, 9-cm Petri dish filled with 30-ml Gamborg's B5 medium (Supplementary Figure 1a). The 9-cm Petri dish was populated by $0,5,15,30$ or 45 seedlings (emitters). For the ACC treatment, Gamborg's B5 medium in the 9-cm Petri dish (emitter medium) was supplemented with $15 \mu \mathrm{g}$ ACC (Sigma, Taufkirchen, Germany). For the $\mathrm{KMnO}_{4}$ treatment, $50 \mathrm{~g} \mathrm{KMnO}_{4}$ beads (Profresh, Bioconservation, Spain) were placed in a 14-cm Petri dish surrounding the 9-cm Petri dish that was placed in the middle (Supplementary Figure 1b). All experiments were performed in growth chambers maintained at $26^{\circ} \mathrm{C}$ with $16 / 8 \mathrm{~h} \mathrm{light/dark} \mathrm{cycle} \mathrm{of}$ $220 \mu \mathrm{mol} / \mathrm{s}$ per $\mathrm{m}^{2}$. Root and hypocotyl lengths of both emitters and receivers were measured 10 days after sowing. Five replicates were used in all experiments whereby the replicated unit was one arena for each treatment.

\section{Ethylene measurements}

Ethylene was measured with a photo-acoustic spectrometer (INVIVO, Adelzhausen, Germany) as described in von Dahl et al. (2007). Seedlings were grown for 10 days and the ethylene in the $250 \mathrm{ml}$ headspace was measured from chambers with: (1) WT seedlings at different densities $(0,5$, 15, 30 and 45); (2) 15 WT or ir-aco seedlings and (3) 15 WT seedlings grown on media supplemented with ACC or in the presence of $\mathrm{KMnO}_{4}$ beads. Five replicate chambers were used in all experiments.

\section{Statistical analyses}

We conducted a linear regression analysis between the number of emitters $(0,5,15,30$ or 45 seedlings) and root growth of WT receivers (SPSS 1999). One-way ANOVAs were carried out to estimate the effect of genotype (WT or ir-aco) or treatments $\left(\mathrm{ACC}\right.$ or $\left.\mathrm{KMnO}_{4}\right)$ on seedling performance. Differences among treatments were analyzed by a post-ANOVA Tukey HSD test and independent sample $t$-test (SPSS 1999). 


\section{Results}

Increased number of emitters reduces root growth of receiver seedlings sharing the same headspace

We used growth arenas in which emitter and receiver seedlings only share a common headspace (Supplementary Figure 1a) and found that increased number of emitters inhibited root growth of WT receiver seedlings $\left(F_{4,116}=\right.$ 12.05, $P<0.0001$; Fig. 1). The negative correlation between number of emitters and the root length of WT receivers (Pearson coefficient $=-0.468, P<0.0001$ ) could only have resulted from sharing a common headspace. Ethylene is a volatile plant hormone that is known to influence plant growth. Analysis of the ethylene concentration in the headspace revealed that its concentration increased with the abundance of emitters (Fig. 1, inset; Pearson coefficient $=0.898$, $P<0.0001)$. Ethylene levels emitted by five WT seedlings were significantly lower than those emitted by 15 $(P=0.028), 30(P=0.004)$ or $45(P<0.0001)$ WT seedlings. The quantities of ethylene in the headspace of five WT seedlings were at the detection limit and did not differ significantly $(P=0.876)$ from the background levels (Fig. 1, inset).

Altered ethylene concentrations in the headspace of emitters effect root growth of WT receivers

Since the correlation between root growth inhibition and ethylene concentration was compelling, we specifically

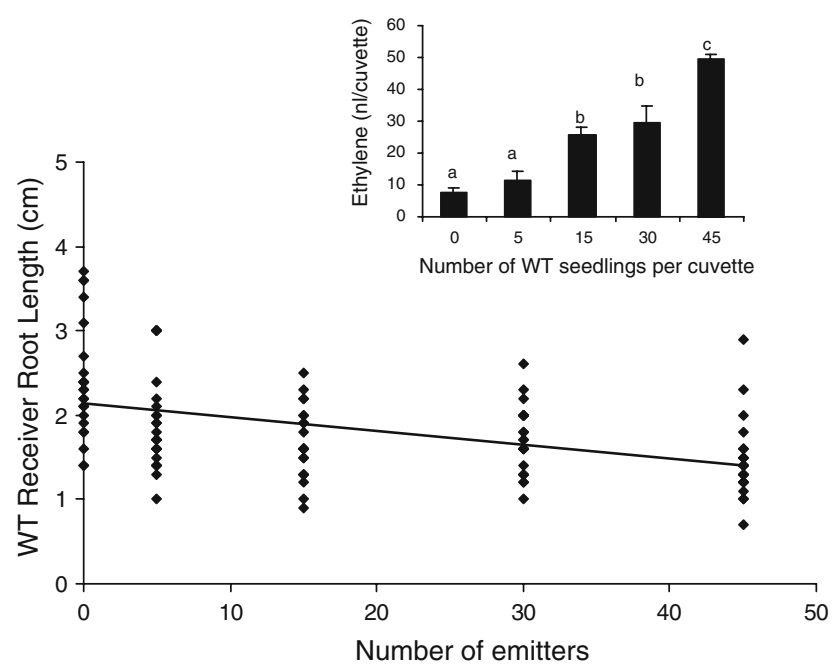

Fig. 1 Regression analysis of root length $(\mathrm{cm})$ of wild-type (WT) Nicotiana attenuata receivers $\left(y=-0.017 x+2.14, \quad R^{2}=0.219\right.$, $P<0.001)$ with a fixed density of five seedlings grown with an increasing number of $0,5,15,30$ and 45 seedlings of WT emitters. Inset: Mean (+SE) ethylene emission (nl/cuvette) from 0, 5, 15, 30 and 45 WT seedlings. Shared letters indicate no significant differences among means as determined by ANOVA with density as fixed variable, and post-ANOVA Tukey tests $(P<0.001)$ enhanced the ethylene emission of emitter seedlings by supplementing the seedlings with ACC, a biosynthetic precursor of ethylene, and reducing ethylene concentrations in the growth arena with the ethylene scrubber $\mathrm{KMnO}_{4}$. Root growth of WT receivers grown in the presence of WT emitters was significantly inhibited compared to the absence of any emitters $(P<0.0001)$, which was also the case in the presence of ACC ( $P=0.007$; Fig. 2). Surprisingly, ACC supplementation did not significantly $\left(t=1.518, P_{2 \text {-tailed }}=\right.$ 0.137 ) inhibit the root growth of WT receivers in the presence of WT emitters (Fig. 2), although the emission of ethylene by the emitter seedlings was significantly enhanced from $38.18 \pm 19.09 \mathrm{nl} / 15$ seedlings/cuvette to $993.8 \pm 217.2 \mathrm{nl} / 15$ seedlings/cuvette $(P<0.0001$; Supplementary Figure 2a).

$\mathrm{KMnO}_{4}$ significantly enhanced the root growth $(\sim 38 \%)$ of WT receivers grown in the presence of WT emitters $\left(t=-3.333, P_{2 \text {-tailed }}=0.002\right)$ compared to the root growth of WT receivers grown in the presence of WT emitters without $\mathrm{KMnO}_{4}$ (Fig. 2). $\mathrm{KMnO}_{4}$ reduced the ethylene concentration to $21.42 \pm 9.22 \mathrm{nl} / 15$ seedlings/cuvette (Supplementary Figure 2b). The effectiveness of the $\mathrm{KMnO}_{4}$ treatment was further demonstrated by its ability to reverse the growth inhibition of receiver roots observed when WT emitters were grown on medium supplemented with ACC (Supplementary Figure 3).

Natural ethylene emissions from emitter seedlings are sufficient to influence the root growth of receivers

In the previous experiments, the relatively small reductions in ethylene concentrations associated with the $\mathrm{KMnO}_{4}$ treatment had a stronger effect on the root growth of receiver seedlings than the large increases in ethylene that

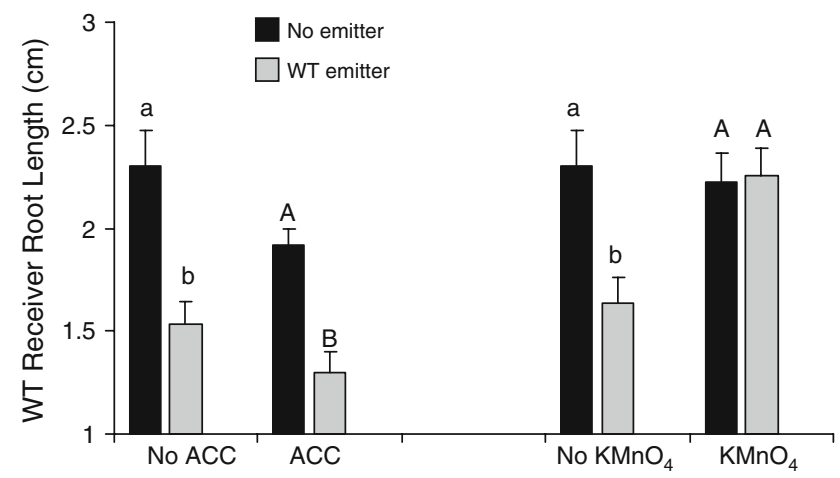

Fig. 2 Mean (+SE) cm root length of wild-type (WT) Nicotiana attenuata receiver seedlings that were grown on media supplemented with 1-aminocyclopropane-1-carboxylic acid (ACC) or in the presence of ethylene scrubbing $\mathrm{KMnO}_{4}$ beads. Five WT receiver seedlings were grown in growth arenas and shared the headspace with $15 \mathrm{WT}$ emitter seedlings. Shared letters indicate no significant differences between means as determined by $t$-test, $P<0.05$ 
resulted from ACC supplementation. To understand whether or not the growth promotion by $\mathrm{KMnO}_{4}$ could be attributed to a reduction in ethylene, we used ir-aco seedlings as emitters and receivers in the analysis. Interestingly, the root growth of WT receivers did not differ in the presence of WT or ir-aco emitters (WT, $P=0.003$; ir-aco, $P=0.032)$ but the growth of ir-aco receiver roots was inhibited in the presence of WT emitters $(P=0.001)$ but not in the presence of ir-aco emitters $(P=0.239)$ (Fig. 3). These differences correlated with significantly higher ethylene emission of WT plants compared to ethylene emitted from ir-aco plants $(P=0.048 ;$ Fig. 3 , inset $)$ and suggested a greater sensitivity of ir-aco plants to exogenous ethylene.

Reduced ethylene production renders plants more sensitive to the ethylene emissions of neighbors

To further explore the sensitivity of ir-aco seedlings, we combined the use of different genotypes and ACC supplementation treatments. ACC supplementation of the growth medium of WT emitter seedling significantly reduced $(\sim 44 \%)$ the root length of ir-aco receivers ( $t=5.88, P_{2 \text {-tailed }}<0.0001$; Fig. 4) but not of WT receivers (Fig. 2). As expected of plants silenced in ACO, ACC supplementation of ir-aco emitters did not have significant ( $\left.t=1.574, P_{2 \text {-tailed }}=0.127\right)$ effects on the root growth of ir-aco receivers (Fig. 4). Furthermore, WT emitters significantly inhibited root growth of ir-aco receivers $(P=0.011)$ compared to that of ir-aco receivers grown without any

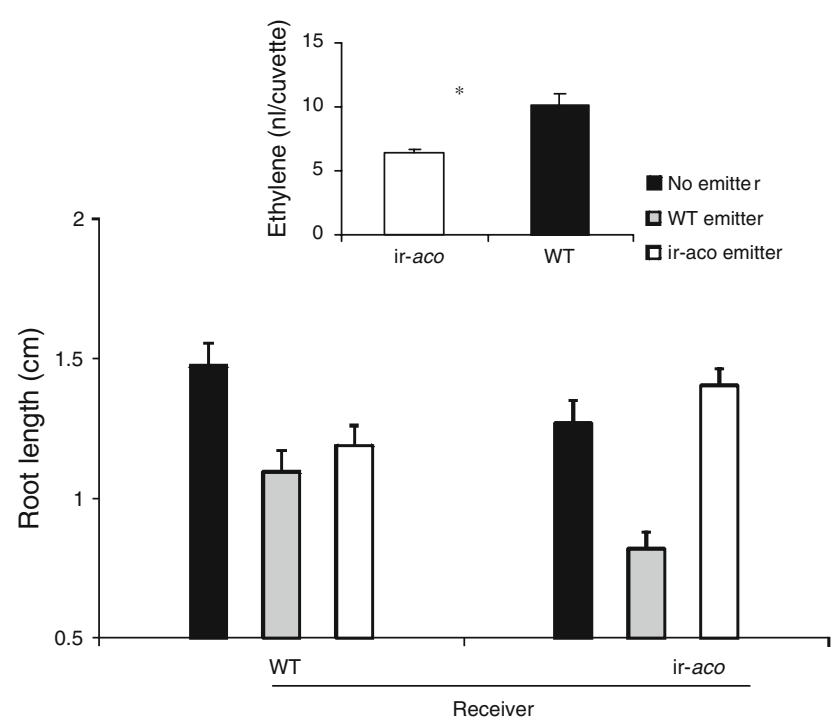

Fig. 3 Mean (+SE) cm root length of wild-type Nicotiana attenuata (WT) and ethylene-silenced ir-aco seedlings. Five receiver seedlings shared the headspace with either no, 15 WT or 15 ir-aco emitter seedlings. Shared letters of the same case indicate no significant differences among means (ANOVA, $P<0.05)$. Inset: Mean (+SE) of ethylene emission (nl/cuvette) from WT and ir-aco seedlings which differed at $P<0.05$

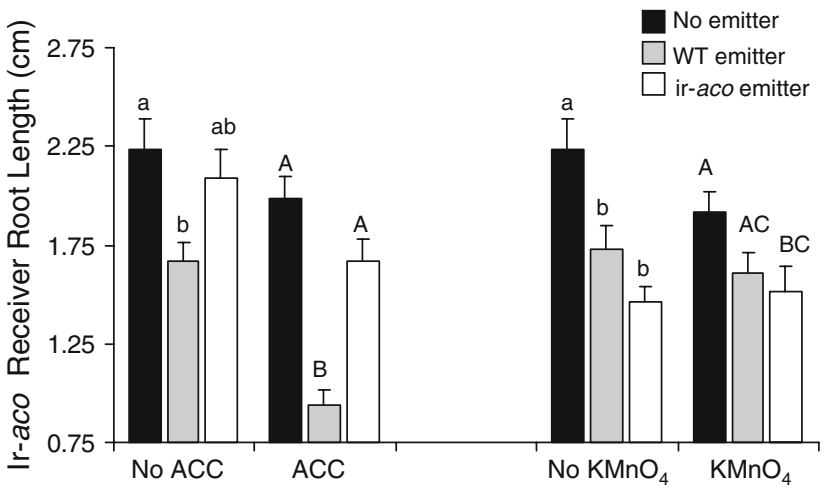

Fig. 4 Mean (+SE) cm root length of ethylene non-producing (ir-aco) Nicotiana attenuata receiver seedlings that were grown on media supplemented with 1-aminocyclopropane-1-carboxylic acid (ACC) or in the presence of ethylene scrubbing $\mathrm{KMnO}_{4}$ beads. Five receiver seedlings were grown in growth arenas and shared the headspace with 15 emitter (WT or ir-aco) seedlings. Shared letters indicate no significant differences among means as determined by ANOVA with treatment as fixed variables, and post-ANOVA Tukey tests; $P<0.05$

emitters but not with ir-aco emitters $(P=0.764$; Fig. 4). No significant $\left(t=0.733, \quad P_{2 \text {-tailed }}=0.467\right)$ difference was observed between root growth of ir-aco receiver grown with WT emitters and $\mathrm{KMnO}_{4}$ and root growth of ir-aco receivers grown with WT emitters but without $\mathrm{KMnO}_{4}$ (Fig. 4).

\section{Discussion}

Ethylene has been studied as a volatile phytohormone in experiments using exogenous applications (Lill et al. 1979; Locke et al. 2000; reviewed by Pierik et al. 2006); here with experiments using WT and transgenic $N$. attenuata seedlings that lack the ability to produce ethylene, we establish that the natural release of ethylene influences the root growth of neighbors. This is the first report demonstrating that the natural release of a volatile chemical inhibits the growth of neighboring siblings, a process called allelopathy. $N$. attenuata is a fire-chaser that germinates in the first growing season after a fire from long-lived seedbanks and therefore frequently initiates growth in dense seedling populations where intense intra-specific competition is the main determinant of Darwinian fitness (Preston and Baldwin 2000). Because of the close proximity of competitors during seedling growth, the demographic importance of seedlings and their sensitivity to naturally released phytohormones, seedling growth is a commonly used parameter for evaluating growth-suppressing activities of phytohormones (Locke et al. 2000). The positive correlation observed between root growth inhibition of WT receivers, the number of WT emitter seedlings that shared the common headspace, and the ethylene levels in the common 
headspace (Fig. 1) is consistent with the hypothesis that the release of ethylene from WT emitters inhibits the root growth of WT receivers. Ethylene accumulation in Petri dishes by $N$. attenuata seedlings is reported to reduce root growth of its seedlings (Hummel et al. 2008). It has also been observed that ethylene, at low, physiologically relevant concentrations, tends to stimulate growth (reviewed in Pierik et al. 2006). However, some studies have shown that ethylene inhibits elongation, but stimulates radial swelling, of roots thereby improving their mechanical strength in resistant soil (Clarke et al. 2003).

Ir-aco emitters produce significantly less ethylene than do WT plants (Fig. 3, inset; von Dahl et al. 2007). We found that WT emitters, which released significantly more ethylene into the headspace, had a much stronger effect on ir-aco receiver seedlings than ir-aco emitters (Fig. 3). The pronounced growth response of ir-aco receivers is consistent with the hypothesized greater ethylene sensitivity of ethylene-silenced ir-aco plants (von Dahl et al. 2007). However, in the absence of any emitter seedlings we did not observe any significant $\left(t=1.783, P_{2 \text {-tailed }}=0.081\right)$ differences between root length of ir-aco and WT seedlings (Fig. 3), which is likely due to the fact that ethylene emitted by five WT seedlings (11.62 $\pm 2.4 \mathrm{nl} /$ cuvette) was not significantly ( $\left.t=1.172, P_{2 \text {-tailed }}=0.280\right)$ different from background emission $(7.87 \pm 1.73 \mathrm{nl} /$ cuvette), thus did not have significant $(P<0.001)$ impact on root growth of WT receivers (see Fig. 1). Furthermore, it is likely that ir-aco plants adapt to their endogenous ethylene concentrations and are able to grow normally in the absence of exogenous ethylene. Relative growth rates of ethylene-insensitive genotypes of Arabidopsis thaliana, Petunia hybrida and Nicotiana tabacum were similar to those of WT growth rates under near optimal growth conditions (Tholen et al. 2004), suggesting that these ethylene-insensitive plants adjusted their growth "normally" in a stress-free environment. Although root lengths of WT receiver was not significantly different when the emitter was WT or ir-aco, the root lengths of WT receivers grown with WT emitters were significantly reduced when compared to no-emitter treatment (Fig. 3). This observation in conjunction with the reduced root lengths of ir-aco receivers grown with WT emitters discussed above establishes that WT emitters influence root growth of receivers (WT or ir-aco) by emitting higher amounts of ethylene compared to ir-aco emitters (Fig. 3, inset). The relative weak silencing of ethylene emission in ir-aco plants, however, may not always have been sufficient to significantly to significantly influence the growth of WT receivers.

The exogenous manipulation of the ethylene concentrations by ACC supplementation and by ethylene scrubbing using $\mathrm{KMnO}_{4}$ only partially supported our hypothesis of ethylene-mediated growth inhibition of WT receivers. Although ACC supplementation of the growth media of
WT emitters resulted in a large ethylene emission $(993.8 \pm$ $217.2 \mathrm{nl} /$ cuvette; Supplementary Figure 2a), only negligible effects on the root growth of WT receiver seedlings was observed (Fig. 2). However, hardly any ethylene was detected from the media $(38.2 \pm 19.1 \mathrm{nl} /$ cuvette $)$ or WT seedlings grown in the presence $\mathrm{KMnO}_{4}(21.4 \pm 9.2 \mathrm{nl} /$ cuvette; Supplementary Figure 2a), and here scrubbing of ethylene by $\mathrm{KMnO}_{4}$ restored the root growth of WT receivers regardless of the presence of WT emitter seedlings (Fig. 2). Surprisingly the effects on ir-aco receivers by these exogenous applications were the opposite and supplementing the growth media of WT emitter seedlings with ACC inhibited the root growth of ir-aco receivers (Fig. 4). These observations reflect the challenges of meaningfully interpreting the effects of high concentration ethylene treatments.

Though various mechanisms for ethylene-mediated root growth suppression are possible, its diffusion through the air from emitters and subsequently through the receiver plant to its root system appears most likely. Root systems are known to be sensitive to volatile and non-volatile chemicals (Inderjit et al. 2005), and ethylene could as well travel through the medium or soil system. A striking example of this sensitivity occurred in the second half of the 20th century when many trees in or near the major cities of Germany died apparently as a result of ethylene leaking from subterranean pipelines (Grümmer 1955).

A series of experiments with cultivated tobacco analyzed the role of ethylene in competition for light and nutrients using transgenic plants over expressing a mutant ethylene receptor (etr1-1) from Arabidopsis thaliana that rendered the plants ethylene insensitive (Pierik et al. 2003, 2004, 2006). These studies elegantly demonstrate that ethylene signaling was required for cultivated tobacco to compete with its neighbors (Pierik et al. 2003), specifically due to its function in the shade-avoidance response of plants (Pierik et al. 2004). However, the responses of the Tetr-1 plants and the WT plants were compared and therefore the experiments could not differentiate between the effects of endogenous ethylene and the potential effects of exogenous ethylene from neighboring plants. In these studies ethylene levels in the air of densely grown cultivated tobacco stands were sufficient to induce growth responses in neighbors. Our study specifically aimed to exclude other factors such as resource competition and shade-avoidance effects and concentrated on the effects of exogenous ethylene. Procedures commonly used in allelopathy research do not isolate the effects of chemicals from other processes that could inhibit the growth of neighbors (Inderjit and Weiner 2001; Lau et al. 2008) and this problem could be resolved by the use of plants that lack the ability to produce a particular chemical.

Transgenics that do not produce a putative allelochemical are excellent tools to determine whether an allelopathic transgenic crop could suppress unwanted neighbors by the 
release of allelochemicals. For example, sorgoleone and $\mathrm{m}$-tyrosine have been suggested as promising candidates for the construction of transgenic allelopathic crops (Bearson et al. 2008; Duke 2007) but conclusive evidence for the allelopathic potential of these compounds in natural environments is lacking. It is important to establish their allelopathic potential before commercializing allelopathic transgenic crops, and we believe that the approach presented in this study can be used to achieve this end.

\section{Conclusion}

Consistent with the hypothesis of ethylene-mediated growth suppression, we found that: (1) headspace ethylene levels were positively correlated with emitter density and negatively correlated with the root growth of receiver seedlings and (2) the inhibition of root growth of WT or ir-aco seedlings by WT emitters was eliminated in the presence of the ethylene scrubber $\mathrm{KMnO}_{4}$ (Jayaraman and Raju 1992) and enhanced by ACC supplementation. From these results, we conclude that the release of a volatile phytohormone or chemical by one plant can influence the growth and establishment of a neighboring conspecific under laboratory conditions. Whether the growth-suppressing activity of ethylene occurs under field situations depends upon the density of the emitter, the accumulation of volatiles at bioactive concentrations and/or climatic conditions.

Acknowledgments We thank A. Weber and A. Schünzel for growing the plants, Harleen Kaur for help in seed germination and preparing growth medium, the Max Planck Society, Indian National Science Academy (INSA) and Deutsche Forschungsgemeinschaft for financial support and two reviewers for constructive comments on an earlier draft of this manuscript.

Open Access This article is distributed under the terms of the Creative Commons Attribution Noncommercial License which permits any noncommercial use, distribution, and reproduction in any medium, provided the original author(s) and source are credited.

\section{References}

Bearson SR, Dayan FE, Rimando AM, Nanayakkara NPD, Liu C, Schroder J, Fishbein M, Pan Z, Kagan IA, Pratt LH, CordonnierPratt MM, Duke SO (2008) A functional genomics investigation of allelochemical biosynthesis in Sorghum bicolor root hairs. J Biol Chem 283:3231-3247

Baldwin IT (2003) Finally, proof of weapons of mass destruction. Sci STKE pe42

Baldwin IT, Halitschke R, Paschold A, von Dahl CC, Preston CA (2006) Volatile signaling in plant-plant interactions: "Talking trees" in the genomics era. Science 311:812-815

Clarke LJ, Whalley WR, Barraclough PB (2003) How do roots penetrate strong soil? Plant Soil 255:93-104

Duke SO (2007) The emergence of grass root chemical ecology. Proc Natl Acad Sci U S A 104:16964-16969
Foo E, Ross JJ, Davies NW, Reid JB, Weller JL (2006) A role for ethylene in the phytochrome-mediated control of vegetative development. Plant J 46:911-921

Gane R (1934) Production of ethylene by some fruits. Nature 134:1008

Grümmer G (1955) Die Gegenseitige Beeinflussung Hoherer PflanzenAllelopahie. Fischer, Jena

Hamilton AJ, Lycett GW, Grierson D (1990) Antisense gene that inhibits synthesis of the hormone ethylene in transgenic plants. Nature 346:284-287

Hummel GM, Schurr U, Baldwin IT, Waltter A (2008) Herbivoreinduced jasmonic acid bursts in leaves of Nicotiana attenuata mediate short-term reductions in root growth. Plant Cell Environ (in press)

Inderjit, Weiner J (2001) Plant allelochemical interference or soil chemical ecology? Perspect Plant Ecol Evol Syst 4:4-12

Inderjit, Saini M, Kaur H (2005) Experimental complexities in evaluating the comparative phytotoxicity of chemicals with different modes of action. Environ Exp Bot 53:97-104

Inderjit, Callaway RM, Vivanco JM (2006) Can plant biochemistry contribute to understanding of invasion ecology? Trends Plant Sci 11:574-580

Jayaraman KS, Raju PS (1992) Development and evaluation of apermanganate-based ethylene scrubber for extending the shelf-life of fresh fruits and vegetables. J Food Sci Technol (Mysore) 29:7783

Karban R (2007) Experimental clipping of sagebrush inhibits seed germination of neighbours. Ecol Lett 10:791-797

Kepczynski J (1986) Inhibition of Amaranthus caudatus seed germination by polyethylene glycol-6000 and abscisic acid and its reversal by ethephon or 1-aminocyclopropane-1-carboxylic acid. Physiol Plant 67:588-591

Klee HJ (2004) Ethylene signal transduction. Moving beyond Arabidopsis. Plant Physiol 135:660-667

Knoester M, van Loon LC, van den Heuvel J, Hennig J, Bol JF, Linthorst HJM (1998) Ethylene-insensitive tobacco lacks nonhost resistance against soil-borne fungi. Proc Natl Acad Sci USA 95:1933-1937

Krügel T, Lim M, Gase K, Halitschke R, Baldwin IT (2002) Agrobacterium-mediated transformation of Nicotiana attenuata, a model ecological expression system. Chemoecology 12:177-183

Lau JA, Puliafico KP, Kopshever JA, Steltzer H, Jarvis EP, Schwarzknder M, Strauss SY, Hufbauer RA (2008) Inference of allelopathy is complicated by effects of activated carbon on plant growth. New Phytol 178:412-423

Lill RE, McWha JA, Cole ALJ (1979) Influence of volatile substances from incubated litter of Pinus radiata on seed germination. Ann Bot 43:81-85

Locke JM, Bryce JH, Morris PC (2000) Contrasting effects of ethylene perception and biosynthesis inhibitors on germination and seedling growth of barley (Hordeum vulgare L.). J Exp Bot 51:18431849

Pierik R, Visser EJW, De Kroon H, Voesenek L (2003) Ethylene is required in tobacco to successfully compete with proximate neighbours. Plant Cell Environ 26:1229-1234

Pierik R, Whitelam GC, Voesenek L, de Kroon H, Visser EJW (2004) Canopy studies on ethylene-insensitive tobacco identify ethylene as a novel element in blue light and plant-plant signalling. Plant $\mathrm{J}$ 38:310-319

Pierik R, Tholen D, Poorter H, Visser EJW, Voesenek L (2006) The Janus face of ethylene: growth inhibition and stimulation. Trends Plant Sci 11:176-183

Preston CA, Baldwin IT (2000) Positive and negative signals regulate germination in the post-fire annual, Nicotiana attenuata. Ecology 81:293-293

Inc SPSS (1999) SPSS Base 10.0 for windows user's guide. SPSS Inc., Chicago 
Tholen D, Voesenek LACJ, Poorter H (2004) Ethylene insensitivity does not increase leaf area or relative growth rate in Arabidopsis, Nicotiana tabacum, and Petunia $x$ hybrida. Plant Physiol 134:1803-1812

von Dahl CC, Winz RA, Halitschke R, Kuhnemann F, Gase K, Baldwin IT (2007) Tuning the herbivore-induced ethylene burst: the role of transcript accumulation and ethylene perception in Nicotiana attenuata. Plant J 51:293-307

Wang KLC, Li H, Ecker JR (2002) Ethylene biosynthesis and signaling networks. Plant Cell 14:S131-S151 